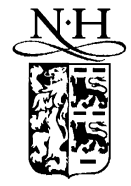

ELSEVIER

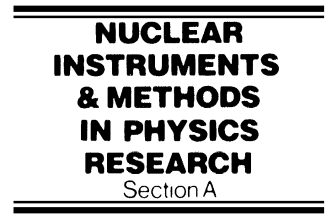

\title{
An ellipsoidal grid gas proportional scintillation counter
}

\author{
Rui M.C. Silva ${ }^{\mathrm{a}, \mathrm{b}}$, J.M.F. dos Santos ${ }^{\mathrm{b}}$, C.A.N. Conde ${ }^{\mathrm{b}, *}$ \\ a Astronomical Observatory, University of Coimbra, Santa Clara P-3040, Coimbra, Portugal \\ ${ }^{\mathrm{b}}$ Physics Department, University of Coimbra, P-3000 Coimbra, Portugal
}

\begin{abstract}
Gas proportional scintillation counters using curved grids for solid angle and reflection compensation have been described in the recent literature. They allow large radiation windows with diameters of $25 \mathrm{~mm}$ keeping at the same time the good energy resolutions characteristic of those X-ray detectors. However, the grids used have a spherical curvature, which does not correspond to the optimal curvature. In the present work we have calculated by computer simulation an improved shape for the curved grid. This shape can be well fitted to an ellipsoid of revolution, with a large eccentricity. A detector was designed with such an ellipsoidal grid and a radiation window $40 \mathrm{~mm}$ in diameter, filled with pure xenon at 927 Torr coupled to an EMI D676QB VUV photomultiplier tube having a 2" diameter window. For the experiments envisaged, detection of solar X-rays in the $20-80 \mathrm{keV}$ energy range, a $7 \mathrm{~cm}$ thick drift region was used, leading to efficiencies from $80 \%$ to $20 \%$, respectively. Such a thick drift region reduces the performance mainly for soft X-rays. For $22 \mathrm{keV}$ X-rays the energy resolution obtained, for a broad X-ray beam entering the full $40 \mathrm{~mm}$ diameter detector window, is $6.0 \%$. Results are presented showing the variation of the energy resolution with the window diameter and a performance, for ellipsoidal grids superior to that for spherical grids. A discussion of the results obtained is presented. (C) 1999 Elsevier Science B.V. All rights reserved.
\end{abstract}

Keywords: Scintillation counters; X-ray detectors; Ellipsoid; Curved grid technique

\section{Introduction}

The demand for large area X-ray detectors having good energy resolution and working at room temperature in fields like X-ray astronomy, medical physics, particle physics, etc., has led to the development of Gas Proportional Scintillation Counters (GPSC) using new techniques like the curved grid one $[1,2]$. With these techniques the variation of

*Corresponding author. Tel.: + 35139410654 . the pulse amplitude with radial distance due to solid angle and reflection effects, is compensated with a radially increasing electric field produced between a plane and a properly shaped curve grid. The results already published $[1,3,4]$ concern only grids with a spherical curvature. While such curved grids give already a good improvement in the energy resolution leading to values as low as $8.0 \%$ for $1^{\prime \prime}$ diameter windows and $5.9 \mathrm{keV} \mathrm{X}$-rays, the results obtained do not correspond yet to the optimum curvature and it seems that there is margin for improvement, since the condition for proper 
compensation [1,2] was only approximately fulfilled. This condition implies that the following integral remains constant as the radial distance, $r$, varies from zero to a limiting value.

$I(r)=\int_{V_{1}}^{V_{2}} \frac{\mathrm{d} n}{\mathrm{~d} s} \Omega(s) R(s) \mathrm{d} s$.

This integral is calculated along the path, $s$, of an electric field line starting in a point of the curved grid at a potential $V_{1}$ and ending in the corresponding point in the other grid at potential $V_{2}$. In the above equation $\mathrm{d} n / \mathrm{d} s$ is the number of scintillation photons, $n$, produced per unit of drifting path length by a single primary electron, $\Omega(s)$ is the solid angle by which the electron views the photomultiplier and $R(s)$ the average reflectivity of the photomultiplier window as seen from the point with coordinate $s$.

Since, as said before, the spherical grid is not the optimum shape, we carried out the present work in search for an improved shape. As it will be shown below, the performance of a gas proportional scintillation counter can be further improved through the use of a grid with an ellipsoid of revolution shape.

\section{The improved curved grid shape}

As has been discussed before [3] the proper shape of the curved grid should be such that the path integral for the light collected by the photocathode and produced along any electric field line (Eq. (1)) remains constant as the radial distance $r$ varies (Fig. 1), which implies a variable distance, $d$, between the grids along a line parallel to the axis.

Since this problem has no obvious analytical solution one way to determine the proper shape could be based in the calculation of that integral using the Monte Carlo method. To simplify the calculations we will assume that the electric field lines are straight lines, between the two grids, parallel to the axis.

We start the calculations by considering the point of the first grid in the detector axis $(r=0)$ and calculate, by the Monte Carlo method, the integral

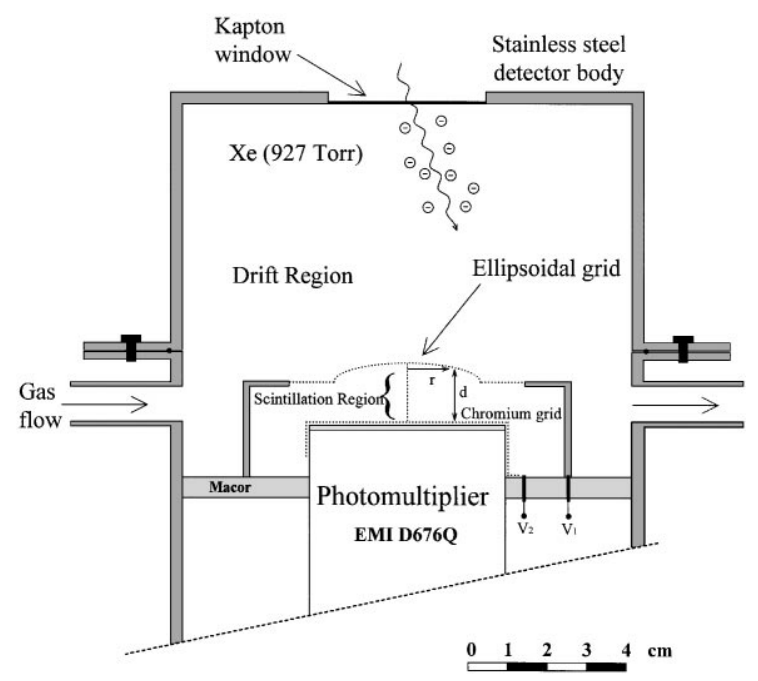

Fig. 1. Gas Proportional Scintillation Counter with an ellipsoidal grid. The ellipse axis are $a=19 \mathrm{~mm}$ and $b=5 \mathrm{~mm}$.

$I(r=0)$ for the case where the planar grid is at a distance $d=d_{0}$ from that point. Then, we consider another point at a distance $r$ from the axis and compute $I(r)$ for a set of values of the distance $d$, with constant voltage difference, $V_{1}-V_{2}$, and take as the best value for $d(r)$ the one that gives a value for $I(r)$ closer to the value previously obtained, $I(r=0)$. The process is repeated for other values of $r$ until a maximum value, $r_{\max }$, and so a set of $d(r)$ values is obtained.

The set of $d(r)$ values obtained are well fitted to an ellipse with the major axis perpendicular to the detector axis.

The voltage difference, $V_{1}-V_{2}$, applied to the grids is such that for $r=r_{\max }$ the reduced electric field intensity is below the threshold for ionisation.

We must emphasise that the calculated curve shape compensates only for solid angle and reflection effects for the voltage difference, $V_{1}-V_{2}$, used in the calculations.

\section{Experimental system}

In Fig. 1 we show the detector designed with the cross section of the ellipsoidal grid developed for this work. This grid, an ellipsoid of revolution 
section, was placed at a distance $d_{0}=15 \mathrm{~mm}$ from the second plane chromium grid deposited onto the photomultiplier window. For $r=r_{\max }(19 \mathrm{~mm})$ the distance between the grids is $10 \mathrm{~mm}$.

Thus, the empirical equation for the calculated ellipse referred to the detector axis and the planar grid, leads to a numerical value for $d(r)$, in $\mathrm{mm}$, given by

$d(r)=10+b \sqrt{1-r^{2} / a^{2}}$.

The ellipse defined by this equation has axis: $a=19 \mathrm{~mm}$ and $b=5 \mathrm{~mm}$.

This GPSC was designed to detect solar X-rays in the $20-80 \mathrm{keV}$ energy range in a high altitude balloon experiment. So a very large $40 \mathrm{~mm}$ diameter window is used together with a $70 \mathrm{~mm}$ thick drift region. The detector body is $100 \mathrm{~mm}$ in diameter and is instrumented with an EMI D676Q photomultiplier tube. The detector is filled with pure xenon at 927 Torr.

\section{Experimental results}

In Fig. 2 we present the variation of the pulse amplitude as a function of the radial distance for

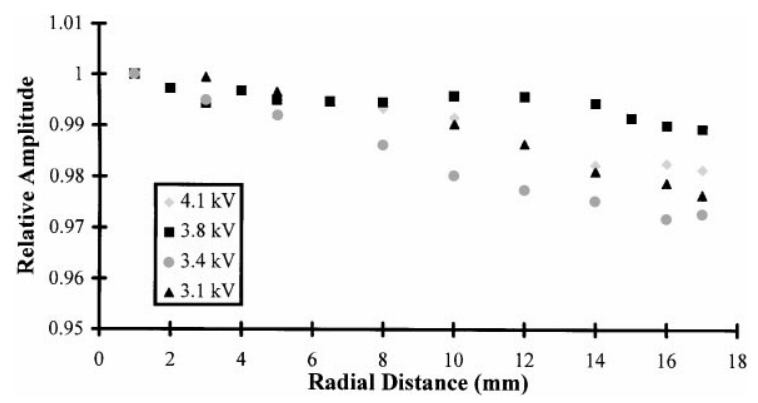

Fig. 2. Relative amplitude as a function of the radial distance for $22 \mathrm{keV} \mathrm{X-rays,} \mathrm{with} \mathrm{an} \mathrm{ellipsoidal} \mathrm{grid,} \mathrm{xenon} \mathrm{filled}$ (927 Torr) gas proportional scintillation counter, for inter-grid voltage of 3.1, 3.4, 3.8 and $4.1 \mathrm{kV}$, showing a more constant pulse amplitude for $3.8 \mathrm{kV}$.

a $22 \mathrm{keV}$ collimated X-ray beam scanning the detector window from $r=0$ to $r=17 \mathrm{~mm}$, for different voltage differences (from 3.1 to $4.1 \mathrm{kV}$ ) across the scintillation region. The pulse amplitude, for an inter-grid voltage of $3.8 \mathrm{kV}$, remains constant within $\pm 0.5 \%$, which means that the solid angle compensation is actually achieved.

In Fig. 3 we present X-ray spectra obtained for two radiation sources: ${ }^{241} \mathrm{Am}$ (main peak

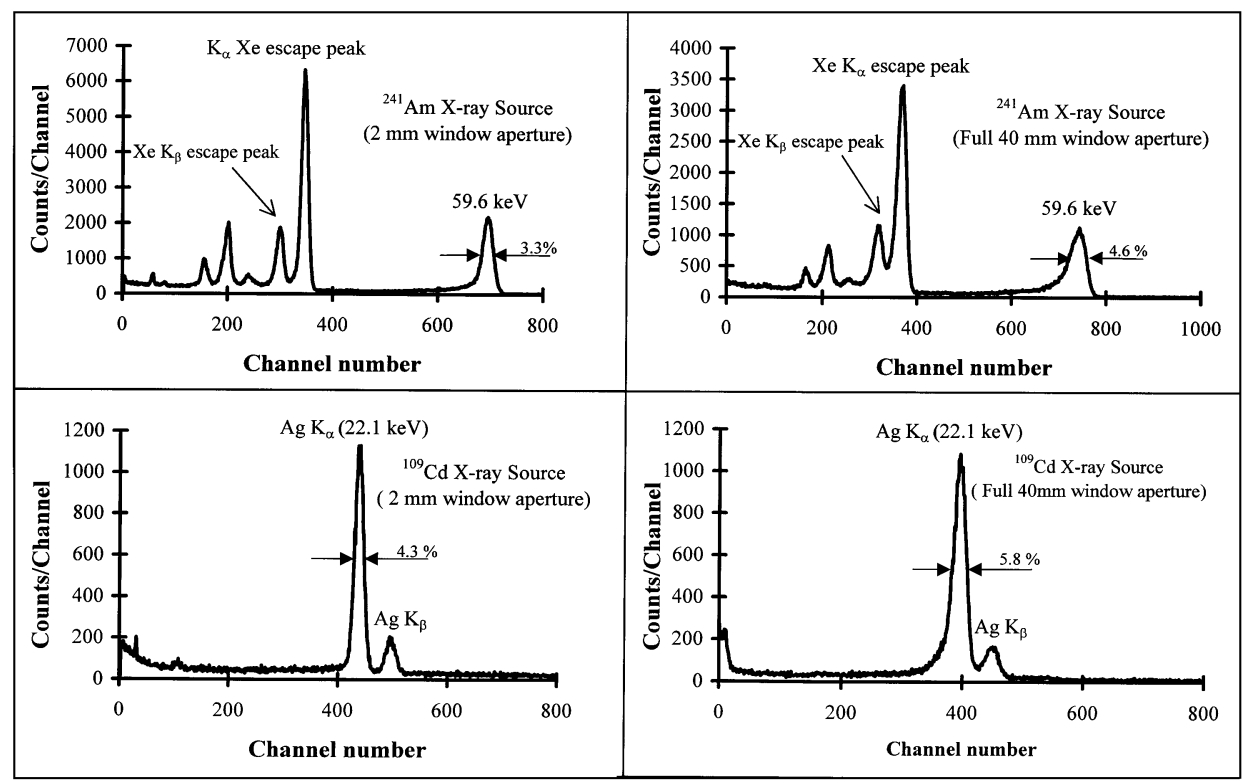

Fig. 3. ${ }^{241} \mathrm{Am}$ and ${ }^{109} \mathrm{Cd} \mathrm{X}$-ray spectra for $2 \mathrm{~mm}$ collimation and full aperture $(40 \mathrm{~mm})$ window. 


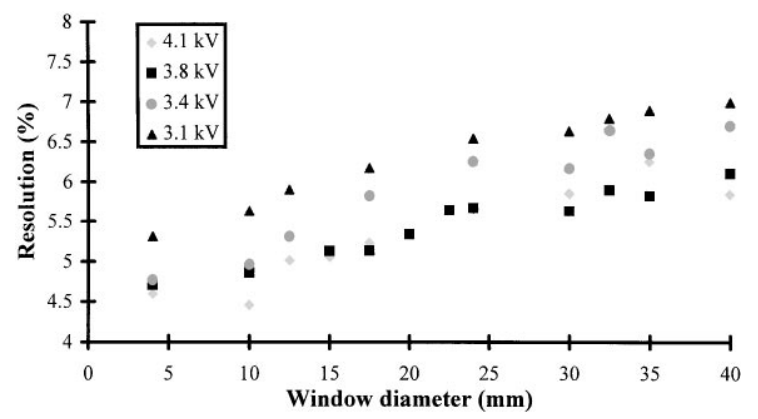

Fig. 4. Energy resolution for ${ }^{109} \mathrm{Cd} \mathrm{X}$-rays $(22 \mathrm{keV})$ as a function of the collimation diameter and the voltage between the grids.

at $59.6 \mathrm{keV}$ ) and ${ }^{109} \mathrm{Cd}$ (main peak at $22.1 \mathrm{keV}$ ), for a centred $2 \mathrm{~mm}$ diameter collimated beam and for a broad beam entering the full $40 \mathrm{~mm}$ diameter window. As shown, the energy resolution only degrades from $4.3 \%$ to $5.8 \%$ when the $22 \mathrm{keV}$ beam diameter increases from 2 to $40 \mathrm{~mm}$. For $59.6 \mathrm{keV}$ the degradation increases from $3.3 \%$ to $4.6 \%$.

In Fig. 4 we present the variation of the energy resolution $(22.1 \mathrm{keV} \mathrm{X}$-rays) for collimation diameters ranging from 4 to $40 \mathrm{~mm}$ and voltage differences between the grids from 3.1 to $4.1 \mathrm{kV}$. As shown the best results are for voltage differences of 3.8 and $4.1 \mathrm{kV}$, in agreement with the results presented in Fig. 2.

\section{Conclusions}

We have shown that ellipsoidal grids for gas proportional scintillation counters provide effective compensation for solid angle and reflection effects, thus allowing detectors with window diameters up to $40 \mathrm{~mm}$. The performance is quite superior to that obtained for parallel grids and the same energy range [5] and better than that obtained for standard proportional counters [6]. The energy resolution of the present design does not degrade for counting rates up to $10^{4}$ counts/s. While the energy resolution is not as good as that obtained with small size $\left(4 \mathrm{~mm}^{2}\right) \mathrm{Cd}_{0.9} \mathrm{Zn}_{0.1} \mathrm{Te}$, cooled $\left(-25^{\circ} \mathrm{C}\right)$ solid state detectors [7] $(1.1 \%$ for $59.6 \mathrm{keV} \mathrm{X}$-rays) its large area $\left(1250 \mathrm{~mm}^{2}\right)$ and room temperature operation are advantageous for many applications like X-ray astronomy. CdTe detectors working at room temperature have also been described [8] but with an energy resolution of $4 \%$ at $59.6 \mathrm{keV}$. Peltier cooled $\mathrm{Si}(\mathrm{Li})$ detectors are commercially available [9] with an energy resolution of $3.2 \%(186 \mathrm{eV})$ at $5.9 \mathrm{keV}$, but the sensitive area is only about $7 \mathrm{~mm}^{2}$ and the shaping time $24 \mu \mathrm{s}$.

While these detectors are not designed for coincidence measurements, timing measurements of the order of a few $\mu$ s are possible.

\section{Acknowledgements}

This work was carried out in the Atomic and Nuclear Instrumentation Group of the Instrumentation Centre (Unit 217/94) of the Physics Department, University of Coimbra. This work was supported by PEDIC/S/FIS/1853/94. Rui M.C. Silva was supported by PRAXIS XXI through the grant PRAXIS/BTI/14504197. Travel support is acknowledged from Gulbenkian Foundation, Lisbon.

\section{References}

[1] C.A.N. Conde, J.M.F. Dos Santos, A.C.S.S.M. Bento, IEEE Trans. Nucl. Sci. NS-40(4) (1993) 452.

[2] C.A.N. Conde, J.M.F. Dos Santos, A.C.S.S.M. Bento, United States Patent, Patent No. 5517030 (May 14, 1996).

[3] J.M.F. Dos Santos, A.C.S.S.M. Bento, C.A.N. Conde, Nucl. Instr. and Meth. A 337 (1994) 427.

[4] L.M.N. Távora, J.M.F. Dos Santos, C.A.N. Conde, SPIE 2280 (1984) 127.

[5] J.M.F. Dos Santos, J.F.C.A. Veloso, R.E. Morgado, C.A.N. Conde, Nucl. Instr. and Meth. A 353 (1994) 195.

[6] H. Sakurai, B.D. Ramsey, M.C. Weiskopf, Nucl. Instr. and Meth. A 313 (1992) 155.

[7] A. Niemela, H. Sipila, V.I. Ivanov, IEEE Trans. Nucl. Sci. NS- 43 (1996) 1476.

[8] A.Kh. Khusainov, Nucl. Instr. and Meth. A 322 (1992) 335.

[9] Amptek Inc., MA, USA (http://www.amptek.com) Model XR-100CR. 